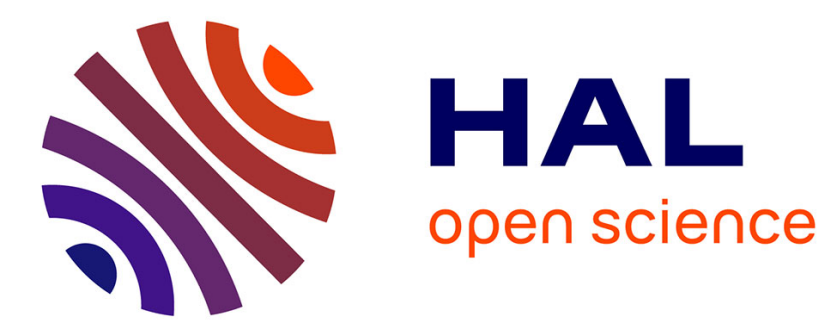

\title{
Discretization of Asymptotically Stable Homogeneous Systems by Explicit and Implicit Euler Methods
}

\author{
Denis Efimov, Andrey Polyakov, Arie Levant, Wilfrid Perruquetti
}

\section{To cite this version:}

Denis Efimov, Andrey Polyakov, Arie Levant, Wilfrid Perruquetti. Discretization of Asymptotically Stable Homogeneous Systems by Explicit and Implicit Euler Methods. Proc. 55th IEEE Conference on Decision and Control (CDC), Dec 2016, Las Vegas, United States. hal-01371275

\section{HAL Id: hal-01371275 \\ https://hal.inria.fr/hal-01371275}

Submitted on 25 Sep 2016

HAL is a multi-disciplinary open access archive for the deposit and dissemination of scientific research documents, whether they are published or not. The documents may come from teaching and research institutions in France or abroad, or from public or private research centers.
L'archive ouverte pluridisciplinaire HAL, est destinée au dépôt et à la diffusion de documents scientifiques de niveau recherche, publiés ou non, émanant des établissements d'enseignement et de recherche français ou étrangers, des laboratoires publics ou privés. 


\title{
Discretization of Asymptotically Stable Homogeneous Systems by Explicit and Implicit Euler Methods
}

\author{
D. Efimov, A. Polyakov, A. Levant, W. Perruquetti
}

\begin{abstract}
Sufficient conditions for the existence and convergence to zero of numeric approximations to solutions of asymptotically stable homogeneous systems are obtained for the explicit and implicit Euler integration schemes. It is shown that the explicit Euler method has certain drawbacks for the global approximation of homogeneous systems with non-zero degrees, whereas the implicit Euler scheme ensures convergence of the approximating solutions to zero.
\end{abstract}

\section{INTRODUCTION}

The problems of stability/performance analysis and control design for continuous-time dynamical systems are very popular and important nowadays. If the system model is linear, then the theory is very-well developed and plenty of approaches exist to solve these problems. In many cases, due to inherited nonlinearity of the plant dynamics, or due to complex quality restrictions imposed on the controlled system, the closed-loop stays nonlinear. Analysis and design methods for such systems are demanded in many applications and are quickly developing the last decades.

Homogeneous dynamical systems become popular since they take an intermediate place between linear and nonlinear systems [1]. They possess some properties of linear ones (e.g. the scalability of trajectories), while being described by essentially nonlinear differential equations, which add such qualities as robustness to measurement noises, exogenous disturbances and delays, or an increased rate of convergence to the goal invariant set.

Frequently for a continuous-time system, after the analysis or design have been performed, for verification or implementation, the system solutions have to be calculated in a computer or in a digital controller (e.g. in a state observer). For these purposes different numerical methods and discretization schemes are used [2]. Since homogeneous systems

All authors are with Non-A team @ Inria, Parc Scientifique de la Haute Borne, 40 avenue Halley, 59650 Villeneuve d'Ascq, France and CRIStAL (UMR-CNRS 9189), Ecole Centrale de Lille, Cité Scientifique, 59651 Villeneuve-d'Ascq, France.

D. Efimov and A. Polyakov are with Department of Control Systems and Informatics, University ITMO, 49 av. Kronverkskiy, 197101 Saint Petersburg, Russia.

A. Levant is with School of Mathematical Sciences,Tel-Aviv University, Tel-Aviv 6997801, Israel.

This work was partially supported by ANR 15 CE23 0007 (Project Finite4SoS), the Government of Russian Federation (Grant 074-U01) and the Ministry of Education and Science of Russian Federation (Project 14.Z50.31.0031). theory is expanding, there are many control or estimation algorithms proposed recently, which possess an increased rate of convergence with respect to linear systems (finite-time or fixed-time convergences [1], [3]), that is why implementation and derivation of solutions for such homogeneous systems need an additional attention.

This paper opens a series of works devoted to application of various discretization schemes for approximation of solutions of homogeneous stable dynamical systems. Different conditions for the existence and convergence to zero of solutions for the explicit and implicit Euler integration schemes are obtained for homogeneous systems. It is shown that application of the explicit Euler method for the global approximation of solutions of homogeneous systems with non-zero degree has some obstructions (see also [4]), and the implicit Euler scheme has a better perspective (see also [5], [6]). However, it is worth to stress that the implicit Euler method has higher computational complexity than the explicit one. Several conditions are proposed, which guarantee existence and convergence to zero of approximations derived by the implicit Euler approach. The obtained results can be taken into account regarding implementations of various fixed-time or finite-time control and estimation algorithms [7], [8], [9], [10], [11], [12], [13], [3].

The outline of this paper is as follows. The notation and preliminary results are introduced in sections II and III. Some basic properties and relations between solution approximations are studied in Section IV. The convergence and divergence conditions are established in Section V. Some simple illustrating examples are considered in Section VI.

\section{NOTATION}

Through the paper the following notation is used:

- $\mathbb{R}_{+}=\{x \in \mathbb{R}: x \geq 0\}$, where $\mathbb{R}$ is the set of real number.

- $|\cdot|$ denotes the absolute value in $\mathbb{R},\|$.$\| denotes the$ Euclidean norm on $\mathbb{R}^{n}$.

- A continuous function $\alpha: \mathbb{R}_{+} \rightarrow \mathbb{R}_{+}$belongs to the class $\mathcal{K}$ if $\alpha(0)=0$ and the function is strictly increasing. The function $\alpha: \mathbb{R}_{+} \rightarrow \mathbb{R}_{+}$belongs to the class $\mathcal{K}_{\infty}$ if $\alpha \in \mathcal{K}$ and it is increasing to infinity.

- The identity matrix of dimension $n \times n$ is denoted as $I_{n}$, and $\operatorname{diag}\left\{r_{i}\right\}_{i=1}^{n}$ is a diagonal matrix with the elements on the main diagonal equal $r_{i}$. 
- A sequence of integers $1,2, \ldots, n$ is denoted by $\overline{1, n}$.

\section{Preliminaries}

In this work the following nonlinear system is considered:

$$
\dot{x}(t)=f(x(t)), t \geq 0,
$$

where $x(t) \in \mathbb{R}^{n}$ is the state, $f: \mathbb{R}^{n} \rightarrow \mathbb{R}^{n}$ ensures forward existence and uniqueness of the system solutions at least locally, $f(0)=0$. For an initial condition $x_{0} \in \mathbb{R}^{n}$ define the corresponding solution by $X\left(t, x_{0}\right)$ for any $t \geq 0$ for which the solution exists. If $f$ is discontinuous, then the solutions are understood in the Filippov's sense [14].

Following [15], [16], [3], let $\Omega$ be an open neighborhood of the origin in $\mathbb{R}^{n}$.

Definition 1. At the steady state $x=0$ the system (1) is said to be

(a) stable if there exists such $\Omega$ that for any $x_{0} \in \Omega$ the solution $X\left(t, x_{0}\right)$ is defined for all $t \geq 0$, and for any $\epsilon>0$ there is $\delta>0$ such that for any $x_{0} \in \Omega$, if $\left\|x_{0}\right\| \leq \delta$ then $\left\|X\left(t, x_{0}\right)\right\| \leq \epsilon$ for all $t \geq 0$;

(b) asymptotically stable if it is stable and for any $\kappa>0$ and $\epsilon>0$ there exists $T(\kappa, \epsilon) \geq 0$ such that for any $x_{0} \in \Omega$, if $\left\|x_{0}\right\| \leq \kappa$ then $\left\|X\left(t, x_{0}\right)\right\| \leq \epsilon$ for all $t \geq T(\kappa, \epsilon)$;

(c) finite-time stable if it is stable and finite-time converging from $\Omega$, i.e. for any $x_{0} \in \Omega$ there exists $0 \leq T<+\infty$ such that $X\left(t, x_{0}\right)=0$ for all $t \geq T$. The function $T_{0}\left(x_{0}\right)=\inf \left\{T \geq 0: X\left(t, x_{0}\right)=0 \forall t \geq T\right\}$ is called the settling time of the system (1);

(d) fixed-time stable if it is finite-time stable and $\sup _{x_{0} \in \Omega} T_{0}\left(x_{0}\right)<+\infty$.

The set $\Omega$ is called the domain of stability/attraction.

If $\Omega=\mathbb{R}^{n}$, then the corresponding properties are called global stability/asymptotic stability/finite-time/fixed-time stability of (1) at $x=0$.

Similarly, the stability notions can be defined with respect to a compact set, by replacing the distance to the origin in Definition 1 with the distance to an invariant set.

\section{A. Weighted homogeneity}

Following [17], [1], [18], for strictly positive numbers $r_{i}$, $i=\overline{1, n}$ called weights and $\lambda>0$, define:

- the vector of weights $\mathbf{r}=\left(r_{1}, \ldots, r_{n}\right)^{T}, r_{\max }=$ $\max _{1 \leq j \leq n} r_{j}$ and $r_{\min }=\min _{1 \leq j \leq n} r_{j}$;

- the dilation matrix function $\Lambda_{r}(\lambda)=\operatorname{diag}\left\{\lambda^{r_{i}}\right\}_{i=1}^{n}$, note that $\forall x \in \mathbb{R}^{n}$ and $\forall \lambda>0$ we have $\Lambda_{r}(\lambda) x=$ $\left(\lambda^{r_{1}} x_{1}, \ldots, \lambda^{r_{n}} x_{n}\right)^{T}$;

- the r-homogeneous norm $\|x\|_{r}=\left(\sum_{i=1}^{n}\left|x_{i}\right|^{\frac{\rho}{r_{i}}}\right)^{\frac{1}{\rho}}$ for any $x \in \mathbb{R}^{n}$ and $\rho \geq r_{\max }$, then there exist $\underline{\sigma}, \bar{\sigma} \in \mathcal{K}_{\infty}$ such that

$$
\underline{\sigma}\left(\|x\|_{r}\right) \leq\|x\| \leq \bar{\sigma}\left(\|x\|_{r}\right) \quad \forall x \in \mathbb{R}^{n} ;
$$

- the sphere and the ball in the homogeneous norm $S_{r}(\rho)=\left\{x \in \mathbb{R}^{n}:\|x\|_{r}=\rho\right\}$ and $B_{r}(\rho)=\{x \in$ $\left.\mathbb{R}^{n}:\|x\|_{r} \leq \rho\right\}$ for $\rho \geq 0$.
Definition 2. A function $g: \mathbb{R}^{n} \rightarrow \mathbb{R}$ is $\mathbf{r}$-homogeneous with degree $\mu \in \mathbb{R}$ if $\forall x \in \mathbb{R}^{n}$ and $\forall \lambda>0$ we have:

$$
\lambda^{-\mu} g\left(\Lambda_{r}(\lambda) x\right)=g(x) .
$$

A vector field $f: \mathbb{R}^{n} \rightarrow \mathbb{R}^{n}$ is $\mathbf{r}$-homogeneous with degree $\nu \in \mathbb{R}$, with $\nu \geq-r_{\min }$ if $\forall x \in \mathbb{R}^{n}$ and $\forall \lambda>0$ we have:

$$
\lambda^{-\nu} \Lambda_{r}^{-1}(\lambda) f\left(\Lambda_{r}(\lambda) x\right)=f(x),
$$

which is equivalent for $i$-th component of $f$ being a $\mathbf{r}$-homogeneous function of degree $r_{i}+\nu$.

System (1) is $\mathbf{r}$-homogeneous of degree $\nu$ if the vector field $f$ is $\mathbf{r}$-homogeneous of the degree $\nu$.

Theorem 1. [17], [19] For the system (1) with $\mathbf{r}$-homogeneous and continuous function $f$ the following properties are equivalent:

- the system (1) is (locally) asymptotically stable;

- there exists a continuously differentiable $\mathbf{r}-$ homogeneous Lyapunov function $V: \mathbb{R}^{n} \rightarrow \mathbb{R}_{+}$such that

$$
\begin{aligned}
& \alpha_{1}(\|x\|) \leq V(x) \leq \alpha_{2}(\|x\|), L_{f} V(x) \leq-\alpha(\|x\|), \\
& \lambda^{-\mu} V\left(\Lambda_{r}(\lambda) x\right)=V(x), \mu>r_{\max }, \\
& \forall x \in \mathbb{R}^{n} \text { and } \forall \lambda>0 \text {, for some } \alpha_{1}, \alpha_{2} \in \mathcal{K}_{\infty} \text { and } \\
& \alpha \in \mathcal{K} .
\end{aligned}
$$

The requirement on continuity of the function $f$ has been relaxed in [20] (the function $V$ can still be selected smooth).

\section{EULER SCHEMES}

If it comes to calculate solution $X\left(t, x_{0}\right)$ of the system (1) for some initial state $x_{0} \in \mathbb{R}^{n}$, then different discretization schemes are used, where the most popular one is the discretization obtained by applying the Euler method (explicit or implicit) [2]. To this end, define a sequence of time instants $t_{i}=i h$ for $i=0,1, \ldots$ and $h>0$ (the discretization step), and denote by $x_{i}=X\left(t_{i}, x_{0}\right)$ the value of the solution of (1) at the corresponding time instant (i.e. $x_{0}=x\left(t_{0}\right)=x(0)$ ), then its approximation calculated in accordance with the explicit Euler method is given by [2]:

$$
x_{i+1}=x_{i}+h f\left(x_{i}\right)
$$

for $i=0,1, \ldots$, while the approximation calculated by the implicit Euler method comes from [2]:

$$
x_{i+1}=x_{i}+h f\left(x_{i+1}\right)
$$

for $i=0,1, \ldots$ Note that formally a different notation should be used in (2) and (3) for the exact solution $X\left(t_{i}, x_{0}\right)$ and its approximation $x_{i}$, but since in this work the problem of approximation accuracy is not considered, and it is well known fact that with $h \rightarrow 0$ both methods approach the real solution [2], then the same symbol will be used in the sequel, where the problem of convergence to zero of the approximations derived in (2) and (3) is studied for stable and homogeneous system (1): 
Assumption 1. Let (1) be r--homogeneous with a degree $\nu$ and globally asymptotically stable.

To proceed we need to establish some properties of solutions in (2) and (3).

\section{A. Existence of approximations}

Existence of some $x_{i+1} \in \mathbb{R}^{n}$ for any $x_{i} \in \mathbb{R}^{n}$ in the explicit case (2) is straightforward, but it is not the case of (3). From homogeneity property we can obtain the following result.

Proposition 1. Let system (1) be $\mathbf{r}$--homogeneous with a degree $\nu \neq 0$. Let for any $x_{0} \in S_{r}(1)$ and all $h>0$ there exist sequences $\left\{x_{i}\right\}_{i=0}^{\infty}$ obtained by (2) or (3) with initial state $x_{0}$. Then for any discretization step $h^{\prime}>0$ and for any $y_{0} \in \mathbb{R}^{n}$ there exist sequences $\left\{y_{i}\right\}_{i=0}^{\infty}$ generated by (2) or (3) with the step $h^{\prime}$ and the initial state $y_{0}$.

All proofs are excluded due to space limitations.

Thus, if there exist sequences $x_{i}, i \geq 0$ generated by (2) or (3) with initial state $x_{0} \in S_{r}$ (1) for any $h>0$, then some sequences $y_{i}, i \geq 0$ will exist for any $y_{0} \in \mathbb{R}^{n}$ and any step $h>0$, but it is hard to make a conclusion about boundedness or convergence of these sequences $y_{i}, i \geq 0$.

In general case, it is difficult to provide some simple conditions for existence and uniqueness of solution of the equation (3). Homogeneity simplifies some derivations.

Proposition 2. If the function $f: \mathbb{R}^{n} \rightarrow \mathbb{R}^{n}$ is continuously differentiable outside the origin, r--homogeneous of degree $\nu \neq 0$ and there exists $h_{0}>0$ such that $\operatorname{det}\left(I_{n}-h_{0} \frac{\partial f(x)}{\partial x}\right) \neq 0$ for all $x \in \mathbb{R}^{n} \backslash\{0\}$, then for $n \geq 2$ the equation (3) has a solution with respect to $x_{i+1} \in \mathbb{R}^{n}$ for any $x_{i} \in \mathbb{R}^{n}$ and for any $h>0$, additionally, for $n \geq 3$ the solution is unique.

B. Relations between approximations obtained for different steps and initial conditions

The main result is as follows.

Proposition 3. Let system (1) be $\mathbf{r}$--homogeneous with a degree $\nu$. If $\left\{x_{i}\right\}_{i=0}^{\infty}$ is a sequence generated by (2) or (3) with the step $h$ and the initial state $x_{0}$, then for any $\lambda>0, y_{i}=\Lambda_{r}(\lambda) x_{i}$ is a sequence obtained by (2) or (3), respectively, with the step $\lambda^{-\nu} h$ and the initial state $y_{0}=\Lambda_{r}(\lambda) x_{0}$

Note that $y_{i}$ is an approximation of the exact solution $X\left(\lambda^{-\nu} h i, y_{0}\right)$ for shifted instants of time. The following corollaries can be established.

Corollary 1. Let system (1) be r--homogeneous with a degree $\nu=0$. Let for all $x_{0} \in S_{r}(1)$ there exist sequences $\left\{x_{i}\right\}_{i=0}^{\infty}$ obtained by (2) or (3) with the step $h>0$ and the initial state $x_{0}$ possessing one of the following properties:

(a) $\sup _{i>0}\left\|x_{i}\right\|<+\infty$;

(b) $\lim _{i \rightarrow+\infty} x_{i}=0$.
Then for any $y_{0} \in \mathbb{R}^{n}$ there exist sequences $y_{i}, i \geq 0$ generated by (2) or (3) with the step $h$ and the initial state $y_{0}$ possessing the same property.

Corollary 2. Let system (1) be r--homogeneous with a degree $\nu \neq 0$. Let there exist $\rho_{0}>0$ and $h_{0}>0$ such that for any $x_{0} \in S_{r}\left(\rho_{0}\right)$ the sequences $\left\{x_{i}\right\}_{i=0}^{\infty}$ obtained by (2) or (3) with the step $h_{0}$ and the initial state $x_{0}$ possess one of the following properties:

(a) $\sup _{i \geq 0}\left\|x_{i}\right\|<+\infty$;

(b) $\lim _{i \rightarrow+\infty} x_{i}=0$.

Then for any $y_{0} \in \mathbb{R}^{n}$ the sequences obtained by (2) or (3) with the step $h_{0}\left(\frac{\left\|y_{0}\right\|_{r}}{\rho_{0}}\right)^{-\nu}$ and the initial state $y_{0}$ possess the same property.

The results of corollaries 1 and 2 show advantages and limitations of the Euler method application for calculation of solutions of homogeneous systems with different degrees. For the case $\nu=0$ the properties of approximation $x_{i}$ depend on size of the step $h$, while for $\nu \neq 0$ if a scheme provides approximation of solutions for some $h$, then similar properties can be obtained for any initial condition with a properly scaled step $h^{\prime}$.

Corollary 3. Let system (1) be r--homogeneous with a degree $\nu \neq 0$. Let for any $x_{0} \in \mathbb{R}^{n}$ and some $h>0$ there exist sequences $\left\{x_{i}\right\}_{i=0}^{\infty}$ obtained by (2) or (3) with initial state $x_{0}$ possessing one of the following properties:
(a) $\sup _{i>0}\left\|x_{i}\right\|<+\infty$;
(b) $\lim _{i \rightarrow+\infty} x_{i}=0$.

Then for any discretization step $h^{\prime}>0$ and for any $y_{0} \in$ $\mathbb{R}^{n}$ there exist sequences $\left\{y_{i}\right\}_{i=0}^{\infty}$ generated by (2) or (3) with the step $h^{\prime}$ and the initial state $y_{0}$ possessing the same property.

Thus, for $\nu \neq 0$ if a scheme provides approximation of solutions globally for some $h$, then similar properties can be obtained for any step $h^{\prime}$. The latter is impossible in general, thus using only homogeneity a global result for the case $\nu \neq$ 0 cannot be obtained for (2) or (3).

\section{Convergence of Sequences $\left\{x_{i}\right\}_{i=0}^{\infty}$ Generated BY EULER METHODS}

In this section we only study the stability features of $\left\{x_{i}\right\}_{i=0}^{\infty}$. The quality of the corresponding approximations of the continuous-time solutions $X\left(t, x_{0}\right)$ by $\left\{x_{i}\right\}_{i=0}^{\infty}$ is considered in the next section.

According to Theorem 1 (see [19], [20]), under Assumption 1 for the system (1) there is a twice continuously differentiable and r--homogeneous Lyapunov function $V$ : 
$\mathbb{R}^{n} \rightarrow \mathbb{R}_{+}$of degree $\mu>-\nu$ such that

$$
\begin{gathered}
a=-\sup _{\xi \in S_{r}(1)} L_{f} V(\xi)>0, \\
0<b=\sup _{\xi \in B_{r}(1)}\left\|\frac{\partial V(\xi)}{\partial \xi}\right\|<+\infty, \\
c_{1}=\inf _{\xi \in S_{r}(1)} V(\xi), c_{2}=\sup _{\xi \in S_{r}(1)} V(\xi), \\
c_{1}\|x\|_{r}^{\mu} \leq V(x) \leq c_{2}\|x\|_{r}^{\mu} \quad \forall x \in \mathbb{R}^{n} .
\end{gathered}
$$

\section{A. Convergence of explicit Euler scheme (2)}

Let us take the discretization step $h>0$ and consider the behavior of $V$ on the sequence generated by (2). For this purpose define $x_{i}=\Lambda_{r}(\lambda) y_{i}$ with $y_{i} \in S_{r}(1)$ and $\lambda=\left\|x_{i}\right\|_{r}$ :

$$
\begin{gathered}
V\left(x_{i+1}\right)-V\left(x_{i}\right)=V\left(x_{i}+h f\left(x_{i}\right)\right)-V\left(x_{i}\right) \\
=\lambda^{\mu}\left[V\left(y_{i}+\lambda^{\nu} h f\left(y_{i}\right)\right)-V\left(y_{i}\right)\right] \\
=\lambda^{\nu+\mu} h \frac{\partial V(\xi)}{\partial \xi} f\left(y_{i}\right)
\end{gathered}
$$

for $\xi=y_{i}+\lambda^{\nu} \varrho f\left(y_{i}\right)$ with $\varrho \in[0, h]$ and the mean value theorem has been used on the last step. Note that

$$
\begin{gathered}
\underline{\sigma}\left(\|\xi\|_{r}\right) \leq\|\xi\| \leq\left\|y_{i}\right\|+\left\|x_{i}\right\|_{r}^{\nu} \varrho\left\|f\left(y_{i}\right)\right\| \\
\leq \bar{\sigma}(1)+g\left\|x_{i}\right\|_{r}^{\nu} h
\end{gathered}
$$

for $g=\sup _{y \in S_{r}}\|f(y)\|$. Next,

$$
\begin{gathered}
V\left(x_{i+1}\right)-V\left(x_{i}\right)=\lambda^{\nu+\mu} h\left\{\frac{\partial V\left(y_{i}\right)}{\partial y_{i}} f\left(y_{i}\right)\right. \\
\left.+\frac{\partial V(\xi)}{\partial \xi} f\left(y_{i}\right)-\frac{\partial V\left(y_{i}\right)}{\partial y_{i}} f\left(y_{i}\right)\right\} \\
\leq h \lambda^{\nu+\mu}\left\{-a+g\left\|\frac{\partial V(\xi)}{\partial \xi}-\frac{\partial V\left(y_{i}\right)}{\partial y_{i}}\right\|\right\} .
\end{gathered}
$$

Since $\left\|\frac{\partial V(\xi)}{\partial \xi}-\frac{\partial V\left(y_{i}\right)}{\partial y_{i}}\right\| \leq k\left\|\xi-y_{i}\right\|$ where $k>0$ is the Lipschitz constant of $\frac{\partial V(\xi)}{\partial \xi}$ on the set $B_{r}\left(\underline{\sigma}^{-1}\left[\bar{\sigma}(1)+g\left\|x_{i}\right\|_{r}^{\nu} h\right]\right)$ (note that $\xi, y_{i} \in B_{r}\left(\underline{\sigma}^{-1}\left[\bar{\sigma}(1)+g\left\|x_{i}\right\|_{r}^{\nu} h\right]\right)$ ), then

$$
\begin{gathered}
V\left(x_{i+1}\right)-V\left(x_{i}\right) \leq h \lambda^{\nu+\mu}\left\{-a+g k\left\|\xi-y_{i}\right\|\right\} \\
\leq h \lambda^{\nu+\mu}\left\{-a+g k \lambda^{\nu} \varrho\left\|f\left(y_{i}\right)\right\|\right\} \\
\leq h \lambda^{\nu+\mu}\left\{-a+g^{2} k \lambda^{\nu} h\right\} .
\end{gathered}
$$

Therefore, the condition of convergence for (2) is

$$
\lambda^{\nu} h<\frac{a}{g^{2} k},
$$

where in the right-hand side all constants are independent on the discretization approach. If (5) is satisfied, then $V\left(x_{i+1}\right)<$ $V\left(x_{i}\right)$, or $\left\|x_{i+1}\right\|_{r}<\left(c_{1}^{-1} c_{2}\right)^{1 / \mu}\left\|x_{i}\right\|_{r}$. The following results can be easily obtained next.

Theorem 2. Let Assumption 1 be satisfied with $\nu=0$, then there exists the discretization step $h>0$ such that the sequences $\left\{x_{i}\right\}_{i=0}^{\infty}$ obtained by (2) for any initial state $x_{0} \in \mathbb{R}^{n}$ and the step $h$ possess the following properties:

(a) $\left\|x_{i}\right\|_{r}<\gamma\left\|x_{0}\right\|_{r}$ for all $i \geq 0$ for some $\gamma \geq 1$;

(b) $\lim _{i \rightarrow+\infty} x_{i}=0$.
Theorem 3. Let Assumption 1 be satisfied with $\nu<0$, then for any $\rho>0$ there exist the discretization step $h_{\rho}>0$ such that the sequences $\left\{x_{i}\right\}_{i=0}^{\infty}$ obtained by (2) for any initial state $x_{0} \notin B_{r}(\rho)$ with a step $h \leq h_{\rho}$ possess the following properties:

(a) $\left\|x_{i}\right\|_{r}<\gamma\left\|x_{0}\right\|_{r}$ for all $i \geq 0$ for some $\gamma \geq 1$;

(b) there exists $i_{x_{0}}>0$ such that $x_{i_{x_{0}}} \in B_{r}(\rho)$.

As follows from Theorem 3, in the case $\nu<0$ for any $h>0$ the explicit Euler scheme provides for the global convergence into some homogeneous ball $B_{r}(\rho)$, and $\rho \rightarrow 0$ as $h \rightarrow 0$. A similar result from [18] also states that the radius $\rho$ is proportional to $h^{-1 / \nu}$.

Theorem 4. Let Assumption 1 be satisfied with $\nu>0$, then for any $\rho>0$ there exists the discretization step $h_{\rho}>0$ such that the sequences $\left\{x_{i}\right\}_{i=0}^{\infty}$ obtained by (2) for any initial state $x_{0} \in B_{r}(\rho)$ with a step $h \leq h_{\rho}$ possess the following properties:

(a) $\left\|x_{i}\right\|_{r}<\gamma\left\|x_{0}\right\|_{r}$ for all $i \geq 0$ for some $\gamma \geq 1$;

(b) $\lim _{i \rightarrow+\infty} x_{i}=0$.

As follows from Theorem 4, in the case $\nu>0$ for any $h>0$ the explicit Euler scheme provides for the asymptotic convergence to zero in some $B_{r}(\rho)$, and $\rho \rightarrow \infty$ as $h \rightarrow 0$. It can be shown that the radius $\rho$ is proportional to $h^{-1 / \nu}$.

\section{B. Convergence of implicit Euler scheme (3)}

Exactly the same results can be obtained for (3). Defining $x_{i+1}=\Lambda_{r}(\lambda) y_{i+1}$ with $y_{i+1} \in S_{r}(1)$ and $\lambda=\left\|x_{i+1}\right\|_{r}$, we obtain

$$
\begin{gathered}
V\left(x_{i+1}\right)-V\left(x_{i}\right)=V\left(x_{i+1}\right)-V\left(x_{i+1}-h f\left(x_{i+1}\right)\right) \\
=\lambda^{\mu}\left[V\left(y_{i+1}\right)-V\left(y_{i+1}-\lambda^{\nu} h f\left(y_{i+1}\right)\right)\right] \\
=\lambda^{\nu+\mu} h \frac{\partial V(\xi)}{\partial \xi} f\left(y_{i+1}\right)
\end{gathered}
$$

for $\xi=y_{i+1}-\lambda^{\nu} \varrho f\left(y_{i+1}\right)$ with $\varrho \in[0, h]$ after application of the mean value theorem on the last step. Next, similarly

$$
\underline{\sigma}\left(\|\xi\|_{r}\right) \leq \bar{\sigma}(1)+g\left\|x_{i+1}\right\|_{r}^{\nu} h
$$

and

$V\left(x_{i+1}\right)-V\left(x_{i}\right) \leq h \lambda^{\nu+\mu}\left\{-a+g\left\|\frac{\partial V(\xi)}{\partial \xi}-\frac{\partial V\left(y_{i+1}\right)}{\partial y_{i+1}}\right\|\right\}$.

Let $k>0$ be the Lipschitz constant of $\frac{\partial V(\xi)}{\partial \xi}$ on the set $B_{r}\left(\underline{\sigma}^{-1}\left[\bar{\sigma}(1)+g\left\|x_{i+1}\right\|_{r}^{\nu} h\right]\right)$, then as before

$$
V\left(x_{i+1}\right)-V\left(x_{i}\right) \leq h \lambda^{\nu+\mu}\left\{-a+g^{2} k \lambda^{\nu} h\right\},
$$

and (5) is the condition of convergence for (3).

More advantageous conditions can be obtained by imposing some additional but mild restrictions (we also assume that solutions exists, i.e. the conditions of Proposition 2 are satisfied).

Theorem 5. Let Assumption 1 hold, $V: \mathbb{R}^{n} \rightarrow \mathbb{R}_{+}$ be a continuously differentiable $\mathbf{r}$--homogeneous Lyapunov 
function of degree $\mu$ for the system (1). Then for the sequence $\left\{x_{i}\right\}_{i=0}^{\infty}$ generated by the implicit scheme (3) with any step $h>0$ and any $x_{0} \in \mathbb{R}^{n}$, the sequence $\left\{V\left(x_{i}\right)\right\}_{i=1}^{+\infty}$ is monotonously decreasing to zero provided that

$$
\langle x-y, f(x)\rangle \neq\|x-y\| \cdot\|f(x)\|
$$

for $x \neq y$ such that $x, y \in\left\{z \in \mathbb{R}^{n}: V(z)=1\right\}$.

It is easy to show that if the level set of the Lyapunov function $V$ is convex the condition (6) of this theorem holds.

Note that a time-varying step can be used in the theorem conditions.

\section{Divergence of explicit Euler scheme (2)}

A consequence of corollaries 2 and 3 is that the Euler methods cannot be used for approximation of solutions of $\mathbf{r}$ -homogeneous systems (1) for a degree $\nu>0$ far outside and for $\nu<0$ close to the origin. This fact also can be proven rigorously (see also [4]).

Theorem 6. Let Assumption 1 be satisfied with $\nu \neq 0$, then for any $h>0$ there exist $\rho_{h}>0$ and $\gamma_{h}>0$ such that $\left\|x_{i}\right\|_{r}>\gamma_{h}\left\|x_{0}\right\|_{r}$ for all $i \geq 0$ and all sequences $\left\{x_{i}\right\}_{i=0}^{\infty}$ obtained by (2) for $x_{0} \in B_{r}\left(\rho_{h}\right) \backslash\{0\}$ if $\nu<0$ and $x_{0} \notin$ $B_{r}\left(\rho_{h}\right)$ if $\nu>0$.

Remark 1. Note that in the proof of Theorem 6, for the case $\nu>0$, a divergence to infinity is shown for any $h>0$ for all $x_{0} \notin B_{r}\left(\rho_{h}\right)$ with properly selected $\rho_{h}>0$. The divergence rate can be estimated as follows for any $i \geq 0$ :

$$
\left\|x_{i+1}\right\|_{r}>\left(\varsigma \frac{c_{1}}{c_{2}}\right)^{1 / \mu}\left\|x_{i}\right\|_{r}
$$

which is exponential if $\varsigma>\frac{c_{2}}{c_{1}} \geq 1$ (in general, a finite-time escape is possible, but it is not obtained). The result of this theorem can also be reformulated as that for any $\rho_{h}>0$ there exist the discretization step $h>0$ such that the trajectories diverge for $x_{0} \notin B_{r}\left(\rho_{h}\right)$ if $\nu>0$ and for $x_{0} \in B_{r}\left(\rho_{h}\right) \backslash\{0\}$ if $\nu<0$.

Theorems 3, 4 and 6 provide a complete characterization of properties of the explicit Euler method (2): for the case $\nu \neq 0$ the Explicit method cannot be applied globally (for the original system the origin is globally attractive and stable, but for $\left\{x_{i}\right\}_{i=0}^{\infty}$ obtained by (2) these global properties are lost for any $\nu \neq 0$ ). Theorem 5 shows that actually under (6) the implicit Euler method (3) can be used.

\section{EXAMPLES}

The series of obtained mathematical results can be illustrated as follows.

Example 1. Consider a scalar stable linear system

$$
\dot{x}=-x,
$$

which is homogeneous of degree $\nu=0$ for $\mathbf{r}=1$. Its discretizations (2) and (3) can be written as follows:

$$
\begin{gathered}
x_{i+1}=(1-h) x_{i}, \\
x_{i+1}=(1+h)^{-1} x_{i} .
\end{gathered}
$$

Thus, the scheme (3) is always converging in this example (by Theorem 5, since the system has a Lyapunov function $V=x^{2}$ for which the condition (6) is satisfied), but (2) is diverging for any $h>2$ (an illustration for Corollary 1).

Example 2. Consider a scalar stable nonlinear system

$$
\dot{x}=-|x| x,
$$

which is homogeneous of degree $\nu=2>0$ for $\mathbf{r}=1$, with its corresponding discretizations (2) and (3):

$$
\begin{gathered}
x_{i+1}=\left(1-h\left|x_{i}\right|\right) x_{i}, \\
x_{i+1}=\frac{1}{2 h}\left(\sqrt{4 h\left|x_{i}\right|+1}-1\right) \operatorname{sign}\left(x_{i}\right) .
\end{gathered}
$$

Then the explicit scheme (2) is converging for any $h>0$ with $\left|x_{i}\right|<2 h^{-1}$ (theorems 4 and 6), while the implicit one (3) is converging globally (by Theorem 5 with $V=x^{2}$ ).

Example 3. Consider another scalar stable nonlinear system

$$
\dot{x}=-|x|^{0.5} \operatorname{sign}(x),
$$

which is homogeneous of degree $\nu=-0.5<0$ for $\mathbf{r}=1$, with its corresponding discretizations (2) and (3):

$$
\begin{gathered}
x_{i+1}=\left(1-h\left|x_{i}\right|^{-0.5}\right) x_{i}, \\
x_{i+1}=\frac{1}{4}\left(\sqrt{4\left|x_{i}\right|+h^{2}}-h\right)^{2} \operatorname{sign}\left(x_{i}\right) .
\end{gathered}
$$

Then the explicit scheme (2) is converging for any $h>0$ with $\left|x_{i}\right|>0.25 h^{2}$ (theorems 3 and and 6), while the implicit one (3) is converging globally (by Theorem 5 with $V=x^{2}$ ).

\section{CONCLUSIONS}

In this work a set of results has been obtained devoted to application of the explicit and implicit Euler methods for discretization of asymptotically stable homogeneous systems. The paper main contributions can be summarized as follows:

- Basic properties deduced for implicit and explicit Euler methods by homogeneity for different values of homogeneity degree $\nu$ :

- Homogeneity simplifies analysis of properties of the obtained discrete approximations of solutions, and there is a certain scalability between approximations calculated for different initial conditions and discretizations steps (Proposition 3).

- For the case of $\nu=0$ the properties of solution approximations are dependent on the discretization step value, and convergence to zero of the scheme for one value of the step does not imply the same property for another one (Theorem 2). However, verification of a global convergence can be performed on a sphere, if for the given discretization 
step the approximation converges to the origin for all initial conditions on the sphere, then the convergence is preserved for any initial conditions (Corollary 1).

- For the case $\nu \neq 0$, convergence to the origin or boundedness of approximations obtained for all initial conditions on a sphere implies the same property for any initial condition with a properly selected discretization step (Corollary 2).

* In the case of $\nu<0$ it has been proved that the approximations globally converge to some vicinity of the origin (Theorem 3 ). The vicinity contracts to 0 as the Euler step tends to 0 .

* For the case $\nu>0$, it has been proved that for sufficiently small steps the approximations locally converge to zero in some vicinity of the origin (Theorem 4). The vicinity covers all state space as step tends to 0 .

- For the case $\nu \neq 0$ global application of the explicit Euler scheme is troublesome since for any value of the step it becomes unstable for sufficiently small $(\nu<0)$ or big $(\nu>0)$ initial conditions (Theorem 6). Of course, for $\nu<0$ convergence to a vicinity of the origin, which is shrinking when discretization step approaches zero [18], can be accepted in many applications, while global divergence for $\nu>0$ should be strictly avoided.

- For the implicit Euler scheme it has been proved, under an additional mild condition, that solutions always exist for any initial conditions and discretization steps (Proposition 2). In addition, the approximations are converging to zero for any initial conditions and discretization steps if the level set of the Lyapunov function of the system is convex (Theorem 5). However, the implicit Euler method has a higher computational complexity than the explicit method, which is the price to pay for all its advantages. Thus, for $\nu<0$ and for a sufficiently small discretization step the explicit Euler method can be a reliable choice.

- For $\nu<0$ the explicit Euler method can be used outside of a vicinity of the origin and next switching to the implicit Euler methods is reasonable, in order to demonstrate convergence to the origin (initial application of the explicit method is motivated by its lower computational complexity).

Future directions of research will include analysis of applicability of Euler methods for locally homogeneous systems, as well as analysis of properties of other methods for approximation of solutions of homogeneous systems.

\section{REFERENCES}

[1] A. Bacciotti and L. Rosier, Lyapunov Functions and Stability in Control Theory, 2nd ed. Springer, Berlin, 2005.

[2] J. C. Butcher, Numerical Methods for Ordinary Differential Equations. New York: John Wiley \& Sons, 2003.

[3] A. Polyakov, "Nonlinear feedback design for fixed-time stabilization of linear control systems," IEEE Transactions on Automatic Control, vol. 57, no. 8, pp. 2106-2110, 2012.
[4] A. Levant, "On fixed and finite time stability in sliding mode control," in IEEE 52nd Annual Conference on Decision and Control (CDC), 2013, pp. 4260-4265.

[5] V. Acary and B. Brogliato, "Implicit euler numerical simulations of sliding mode systems," System and Control Letters, vol. 59, no. 5, pp. 284-293, 2010.

[6] B. Brogliato and A. Polyakov, "Globally stable implicit euler timediscretization of a nonlinear single-input sliding-mode control system," in IEEE CDC, 2015, pp. 5426-5431.

[7] Y. Hong, J. Huang, and Y. Xu, "On an output feedback finite-time stabilization problem," IEEE Trans. Automat. Control, vol. 46, no. 2, pp. 305-309, 2001.

[8] S. P. Bhat and D. S. Bernstein, "Geometric homogeneity with applications to finite-time stability," Mathematics of Control, Signals and Systems, vol. 17, pp. 101-127, 2005.

[9] J. Li and C. Qian, "Global finite-time stabilization by dynamic output feedback for a class of continuous nonlinear systems," IEEE Transactions on Automatic Control, vol. 51, no. 5, pp. 879-884, 2006.

[10] V. Andrieu, L. Praly, and A. Astolfi, "Homogeneous Approximation, Recursive Observer Design, and Output Feedback," SIAM J. Control Optimization, vol. 47, no. 4, pp. 1814-1850, 2008.

[11] E. Moulay, "Stabilization via homogeneous feedback controls," Automatica, vol. 44, no. 11, pp. 2981-2984, 2008.

[12] W. Perruquetti, T. Floquet, and E. Moulay, "Finite-time observers: application to secure communication," IEEE Transactions on Automatic Control, vol. 53, no. 1, pp. 356-360, 2008.

[13] E. Cruz-Zavala, J. Moreno, and L. Fridman, "Uniform robust exact differentiator," IEEE Transactions on Automatic Control, vol. 56, no. 11 , pp. $2727-2733,2011$.

[14] A. Filippov, Differential Equations with Discontinuous Righthand Sides. Kluwer Academic Publishers, 1988.

[15] E. Roxin, "On finite stability in control systems," Rendiconti del Circolo Matematico di Palermo, vol. 15, pp. 273-283, 1966.

[16] H. K. Khalil, Nonlinear Systems, ser. NJ 07458. Upper Saddle River: Prentice-Hall, 1996.

[17] V. Zubov, "On systems of ordinary differential equations with generalized homogenous right-hand sides," Izvestia vuzov. Mathematica., vol. 1, pp. 80-88, 1958 (in Russian).

[18] A. Levant, "Homogeneity approach to high-order sliding mode design," Automatica, vol. 41, no. 5, pp. 823-830, 2005.

[19] L. Rosier, "Homogeneous Lyapunov function for homogeneous continuous vector field," Systems \& Control Letters, vol. 19, pp. 467-473, 1992.

[20] E. Bernuau, A. Polyakov, D. Efimov, and W. Perruquetti, "On extension of homogeneity notion for differential inclusions," in Proceeding of the European Control Conference, Zurich, 2013, pp. 2204-2209. 